\title{
Detailed Modeling and Analysis of Spacecraft Plume/Ionosphere Interactions in Low Earth Orbit
}

\author{
Kelly A. Stephani* and Iain D. Boyd ${ }^{\dagger}$ \\ Department of Aerospace Engineering, University of Michigan, Ann Arbor, MI, 48109, USA
}

\begin{abstract}
Detailed direct simulation Monte Carlo/Particle in Cell simulations involving the interaction of spacecraft thruster plumes with the rarefied ambient ionosphere are presented for steady thruster firings in Low Earth Orbit (LEO). A nominal mass flow rate is used to prescribe the rocket exit conditions of a neutral propellant species for use in the simulations. The charge exchange interactions of the steady plume with the rarefied ionosphere are modeled using a direct simulation Monte Carlo/Particle in Cell methodology, allowing for a detailed assessment of non-equilibrium collisional and plasma-related phenomena relevant for these conditions. Results are presented for both ram- and wake-flow configurations, in which the thrusters are firing into (ram) or in the direction of (wake) the free stream ionosphere flow in LEO. The influence of the Earth's magnetic field on the development of the ion plume is also examined for three different field strengths: two limiting cases in which $B \rightarrow 0$ and $B \rightarrow \infty$, and the LEO case in which $B=0.5$ Gs. The magnetic field is found to have a substantial impact on the resulting neutral and ion plumes, and the gyroscopic motion of the magnetized ions results in a broadening of the ion energy distribution functions. The magnetic field model also incorporates a cross-field diffusion mechanism which is shown to increase the current density sampled far from the thruster origin.
\end{abstract}

\section{Introduction}

A fundamental understanding of plume dynamics is imperative for developing both predictive and mitigatory capabilities to avoid plume impingement on critical spacecraft surfaces. Chemical interactions between post-combustion neutral species generated by spacecraft thrusters and ambient ions in the upper atmosphere play an important role in determining the dynamic behavior of these plumes. In particular, the high-density neutral plume emitted during a thruster burn is subject to charge exchange reactions with the ambient ions. This interaction can alter the local ionospheric properties, and lead to excitation of plasma waves. Studies of such interactions, both experimental and computational, have been centralized around LEO transportation spacecraft, including Space Shuttle, Soyuz, Progress and the Mir space station. ${ }^{1-6}$

In particular, the study by Burke et al. ${ }^{1}$ examined the energy distribution of positive, single-charge ions detected by the Shuttle Potential and Return Electron Experiment (SPREE) during a thruster burn of the Tethered Satellite System (TSS 1) mission. Data collected by this sensor included information regarding both energy and angular distributions of ions impacting the sensor, over ion energies ranging from 10 to $100 \mathrm{eV}$. This study also compared SPREE data with results from a two-dimensional collisionless molecular model. The model tracked trajectories of neutrals and pick-up ions (plume-related ions formed through charge transfer of plume neutrals with ambient ions) during a thruster burn event, and provided information regarding the distribution of ions that eventually impact the SPREE sensor. This allowed for a comparison between the measured and predicted ion energy distributions. It was also found that significant scattering occurs near the thruster exit as well as after charge exchange between the neutral gas and ambient oxygen ions.

The present study aims to examine the interaction between spacecraft thruster plumes and the ambient ionosphere in Low Earth Orbit conditions. While previous work has examined near-field plume/ ionosphere

${ }^{*}$ Postdoctoral Research Fellow, Department of Aerospace Engineering, 1320 Beal Ave, Ann Arbor, MI, Member AIAA.

$\dagger$ James E. Knott Professor, Department of Aerospace Engineering, 1320 Beal Ave, Ann Arbor, MI, Fellow AIAA. 
interactions, this study will focus on non-equilibrium collisional and plasma-related phenomena over extended distances of many kilometers. The rarefied nature of the ionosphere, as well as the surrounding plasma, requires the use of a combined direct simulation Monte Carlo/Particle in Cell (DSMC/PIC) methodology, which is detailed next. It is assumed that the neutral rocket plume is comprised of a single propellant species. Results are presented for a steady spacecraft thruster burn, with two rocket plume configurations examined. The effects from the geomagnetic field on the development and propagation of the ion plume is also investigated. Specifically, three magnetic field strengths are examined: two limiting cases in which the ion plume develops in the absence of a magnetic field as well as in an infinitely strong magnetic field, and a third intermediate case in which the field strength is equivalent to that at LEO. Finally, conclusions drawn from this study are presented, and direction for future work is discussed.

\section{Modeling of Plume/Ionosphere Interactions}

\section{A. DSMC/PIC Framework}

The charge exchange collisions between ambient ions and rocket plume propellant occur under very low density conditions. The most appropriate numerical method for simulation of these phenomena is the direct simulation Monte Carlo (DSMC) method. ${ }^{7}$ The plasma formed in this process is subject to self-consistent electro-static fields, which is most appropriately modeled using the Particle in Cell (PIC) method. ${ }^{8}$ The combination of rarefied collisional and plasma phenomena relevant to the physical system of interest is therefore analyzed using MPIC, ${ }^{9}$ which uses the DSMC and PIC methods simultaneously to model the flow field.

\section{B. MEX/CEX Collision Dynamics}

The chemical system under consideration is comprised of four chemical species: spacecraft neutrals/ions and ambient (ionosphere) neutrals/ions. The spacecraft thrusters eject a high-density plume of neutral particles, comprised mostly of water vapor, which expands into the surrounding ambient flow. It is assumed that the spacecraft neutral plume constituents are modeled as a single propellant species with a corresponding ion, referred to as $\mathrm{Pr}$ and $\mathrm{Pr}^{+}$. The molecular weight of the $\mathrm{Pr}$ species is equivalent to the molecular weight of the neutral plume mixture, and the collisional properties of $\mathrm{Pr}$ and $\mathrm{Pr}^{+}$follow those of water vapor. The ambient ionosphere model used in this study is comprised of the primary neutral and ion species found at $\mathrm{LEO}, \mathrm{O}$ and $\mathrm{O}^{+}$. Interaction of $\mathrm{Pr}$ with the ambient $\mathrm{O}^{+}$leads to the formation of $\mathrm{Pr}^{+}$through a charge exchange (CEX) reaction.

The $\mathrm{O}^{+}$ions are allowed to participate in both momentum exchange (MEX) and CEX interactions, but the post-collision properties of $\mathrm{O}^{+}$are not updated. As will be discussed later in this section, this effectively models the $\mathrm{O}^{+}$as being trapped indefinitely on the geomagnetic field lines. The neutral $\mathrm{O}$ atoms are allowed to participate in MEX interactions only, but the post-collision properties are updated. This serves to preserve the ratio of ambient $\mathrm{O}^{+}$ions and $\mathrm{O}$ neutrals throughout the computational domain. Both $\mathrm{Pr}$ and $\mathrm{Pr}^{+}$participate in MEX/CEX interactions. A summary of the permitted interactions for this chemical system are provided in Table 1. The rotational and vibrational internal structure of plume constituents (Pr, $\mathrm{Pr}^{+}$) is neglected in this work.

Table 1. Permitted interactions between plume/ambient chemical species

\begin{tabular}{|c|c|c|c|c|}
\hline & $\mathrm{Pr}$ & $\mathrm{Pr}^{+}$ & $\mathrm{O}$ & $\mathrm{O}^{+}$ \\
\hline $\mathrm{Pr}$ & $\mathrm{MEX}$ & $\mathrm{MEX} / \mathrm{CEX}$ & $\mathrm{MEX}$ & $\mathrm{MEX} / \mathrm{CEX}$ \\
$\mathrm{Pr}^{+}$ & & - & $\mathrm{MEX}$ & - \\
$\mathrm{O}$ & & & $\mathrm{MEX}$ & $\mathrm{MEX}$ \\
$\mathrm{O}^{+}$ & & & & - \\
\hline
\end{tabular}

Heavy particle interactions are treated according to standard DSMC collision dynamics, with the possibility of a charge transfer for neutral/ion collision pairs. The total number of candidate collision partners within a cell is determined using Bird's No-Time-Counter (NTC) ${ }^{7}$ method. The probability of a collision event is then determined for these candidate pairs based on the total collision cross section. 


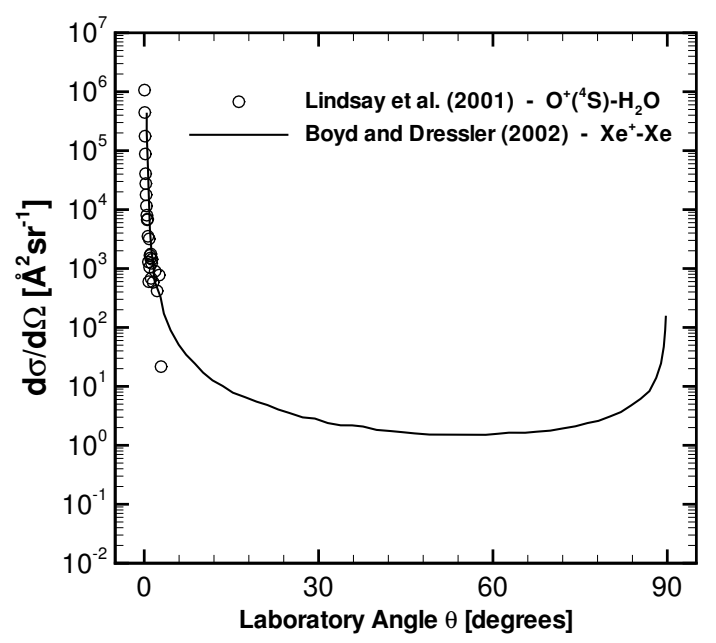

(a) DCS used for $\mathrm{O}^{+}-\operatorname{Pr}$ system.

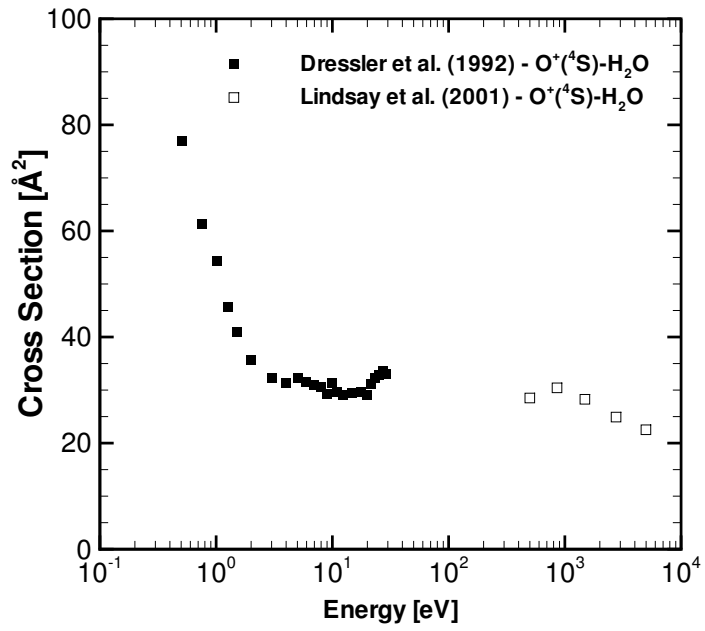

(b) TCS used for $\mathrm{O}^{+}-$Pr system.

Figure 1. (a) Differential cross sections (DCS) and (b) total cross sections (TCS) used for modeling charge exchange collision dynamics of $\mathrm{O}^{+}-\mathrm{Pr}$ system.

Analysis by Boyd and Dressler ${ }^{10}$ and experimental measurements by Pullins et al. ${ }^{11}$ and Miller et al. ${ }^{12}$ demonstrate that the $\mathrm{Xe}^{+}-\mathrm{Xe}$ elastic and charge exchange total cross sections are essentially equivalent, such that $\sigma_{M E X} \approx \sigma_{C E X}$. This allows for the use of a single total cross section (TCS) $\sigma$ and the relative collision velocity, $g$, to determine the probability $P$ of a collision event according to:

$$
P_{M E X}=\frac{\sigma g}{(\sigma g)_{\max }},
$$

where a candidate pair is selected for collision if $P_{M E X}>\mathcal{R}_{u}$. If the collision under consideration involves a neutral/ion pair, the probability of a CEX event is taken as $P=0.5,{ }^{10}$ such that the total probability of a collision resulting in a charge exchange is:

$$
P_{C E X}=P_{M E X} \times P .
$$

This approach is adopted for the $\mathrm{O}^{+}-\mathrm{Pr}$ and $\mathrm{Pr}^{+}-\mathrm{Pr}$ interactions modeled in this chemical system, although the validity of this assumption should be examined through analysis of the differential cross section (DCS) data which are not available for the energy range of interest.

Post-collision velocities involving neutral/neutral collision pairs are assumed to follow isotropic scattering, while collisions involving neutral/ion pairs scatter anisotropically, with a strong forward-scattering tendency. This anisotropic scattering is incorporated into the MPIC CEX model through the use of experimental DCS data. Figure 1(a) presents measurements of the absolute DCS for CEX scattering of $\mathrm{O}^{+}\left({ }^{4} \mathrm{~S}\right)$ with $\mathrm{H}_{2} \mathrm{O}$ at 500 $\mathrm{eV}$ obtained by Lindsay et al. ${ }^{13}$ These measurements were acquired over a limited range of scattering angles, from $0.04-2.9^{\circ}$ in the laboratory frame of reference. While many of the scattered particles were found to lie within this narrow range, a comparison of the DCS, integrated from $0-3.0^{\circ}$, to an estimated TCS indicates that the DCS measurements in Figure 1(a) capture approximately $74 \%$ of the estimated TCS shown in Figure 1(b). Although the energies considered in this work are significantly lower than those presented in Lindsay et al., this appears to be the only differential cross section data available for the $\mathrm{O}^{+}-\mathrm{H}_{2} \mathrm{O}$ system, for either ground state $\mathrm{O}^{+}\left({ }^{4} \mathrm{~S}\right)$ or metastable $\mathrm{O}^{+}\left({ }^{2} \mathrm{D},{ }^{2} \mathrm{P}\right)$.

To use this data within the MPIC framework, the differential cross section data is first converted from a laboratory (LAB) frame of reference to the center-of-mass (COM) frame of reference according to: ${ }^{14}$

$$
\frac{d \sigma}{d \Omega_{L A B}}=\frac{d \sigma}{d \Omega_{C O M}} 4 \cos \theta_{L A B},
$$

where $d \Omega$ is the solid angle and $\theta$ is the scattering angle. The resulting distribution is then normalized by the maximum value, and post-collision scattering angles are thereby sampled. To account for the possibility of 
a large-angle scattering event, the DCS for a $\mathrm{Xe}^{+}-\mathrm{Xe}$ system at $300 \mathrm{eV}$, plotted in Figure 1(a), is used for angles larger than $3^{\circ}$. The probability of a post-collision scattering angle larger than $3^{\circ}$ is less than 0.025 , so inclusion of this data for large scattering angles has a small impact on the post-collision distributions.

As mentioned before, the typical collision energies considered in this work are $O(10) \mathrm{eV}$, which are considerably lower than the energies presented in the total cross section data of Lindsay et al. Fortunately, several measurements of the total cross section for the $\mathrm{O}^{+}\left({ }^{4} \mathrm{~S}\right)-\mathrm{H}_{2} \mathrm{O}$ system are available at lower energy. ${ }^{15-17}$ The total cross section for the $\mathrm{O}^{+}\left({ }^{4} \mathrm{~S}\right)-\mathrm{H}_{2} \mathrm{O}$ system used in this work is fitted from measurements by Dressler et al. and Lindsay et al. shown in Figure 1(b). MEX collisions for $\mathrm{Pr}-\mathrm{O}, \mathrm{O}-\mathrm{O}$ and $\mathrm{Pr}-\mathrm{Pr}$ are modeled using variable hard sphere ${ }^{7}$ (VHS) total cross sections and isotropic scattering. The corresponding VHS parameters including the reference diameter $d_{r e f}$, reference temperature $T_{r e f}$, and temperature exponent $\omega$, are provided in Table 2 .

Table 2. VHS parameters for $\mathrm{Pr}-\mathrm{O}, \mathrm{O}-\mathrm{O}, \mathrm{Pr}-\mathrm{Pr}$

\begin{tabular}{|c|c|c|c|}
\hline & $d_{\text {ref }}$ & $T_{\text {ref }}$ & $\omega$ \\
\hline $\mathrm{Pr}$ & $4.0 \AA$ & $273 \mathrm{~K}$ & 0.75 \\
$\mathrm{O}$ & $3.0 \AA$ & $273 \mathrm{~K}$ & 0.75 \\
\hline
\end{tabular}

\section{Magnetic Field Model}

In addition to CEX interactions, charged particles in LEO are subject to interaction with Earth's magnetic field. The interaction of spacecraft ions with the magnetic field plays an important role in the evolution of the ion plume. Both the strength of the magnetic field and the orientation of the field lines relative to the plume propagation have a substantial impact on the development of the plume, as magnetization of spacecraft ions can impede ion flow in the axial and radial directions. The magnetic field model developed for this work investigates the impact of magnetic field strength on the spacecraft ion plume formation assuming a fixed field line orientation relative to the spacecraft.

Immediately after a CEX event, the newly formed ion enters into a gyroscopic orbit about a magnetic field line. This orbit is characterized by the Larmor radius, $r_{L}$, and the gyration frequency, $\omega_{L}$, which are determined according to:

$$
\begin{gathered}
r_{L}=\frac{V_{x 0}^{P r^{+}}}{\omega_{L}}, \\
\omega_{L}=\frac{q^{P r^{+}} B}{m^{P r^{+}}} .
\end{gathered}
$$

In Eq. $4, V_{x 0}^{P r^{+}}$is the initial x-velocity of the $\mathrm{Pr}^{+}$species entering the gyro-orbit, which is equivalent to the post-collision $\mathrm{x}$-velocity after a charge exchange reaction. In Eq. $5, q^{P r+}$ is the fundamental charge, $B$ is the magnetic field strength, and $m^{P r+}$ is the molecular mass of the $\mathrm{Pr}^{+}$species. From these expressions, it is clear that the magnetic field strength uniquely determines the gyration frequency for a given charged chemical species. The Larmor radius, however, is dependent on both magnetic field strength (through $\omega_{L}$ ) as well as on the translational energy of the magnetized ion orthogonal to the field line.

Within the present axisymmetric simulations, the magnetic field lines are assumed to be oriented vertically (parallel to the $z$-axis, shown by dashed lines in Figure 2). Thus, only the $x$-velocity component of the magnetized ions follows a gyroscopic motion, while the $z$-velocity component is unimpeded by the magnetic field. Magnetized ions entering a gyroscopic orbit with a non-zero velocity component along the field line thus follow a helical trajectory. The frame of reference is held fixed to the spacecraft thruster at the origin, such that the ambient flow, and hence the geomagnetic field lines, have a velocity equivalent to the orbital velocity relative to the spacecraft. The gyroscopic motion due to the magnetic field is imposed on the $\mathrm{Pr}^{+}$ ions through a time-dependent velocity, which for a constant magnetic field aligned with the $z$-axis, is determined according to:

$$
V_{x}^{i o n}=V_{B}-\left(V_{x 0}^{i o n}-V_{B}\right) \sin \left(\frac{q^{i o n} B}{m^{i o n}} t+\phi^{i o n}\right),
$$




$$
\phi^{i o n}= \begin{cases}\pi / 2 & \text { if } V_{x o}^{i o n}<V_{B} \\ 3 \pi / 2 & \text { if } V_{x 0}^{i o n}>V_{B}\end{cases}
$$

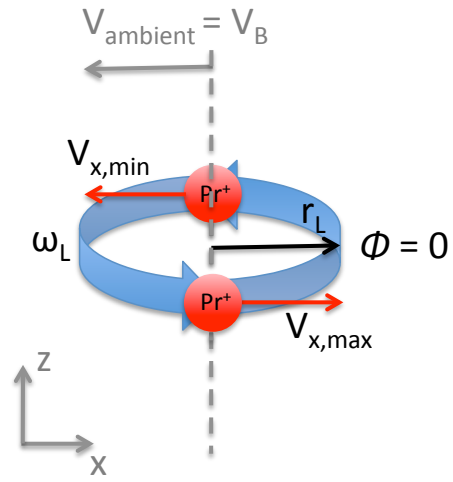

(a) Magnetized $\mathrm{Pr}^{+}$ion.

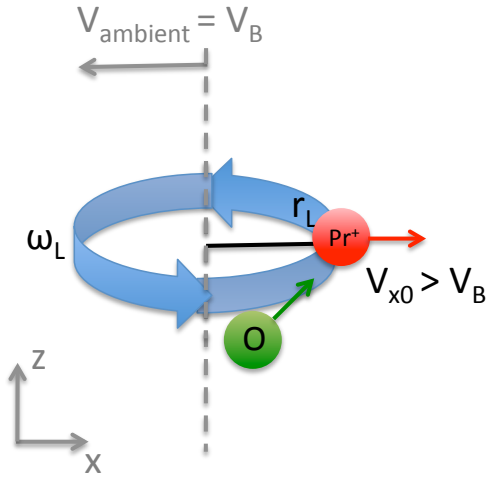

(b) $\mathrm{O}-\mathrm{Pr}^{+}$collision resulting in MEX and new guiding center for $\mathrm{Pr}^{+}$ion.

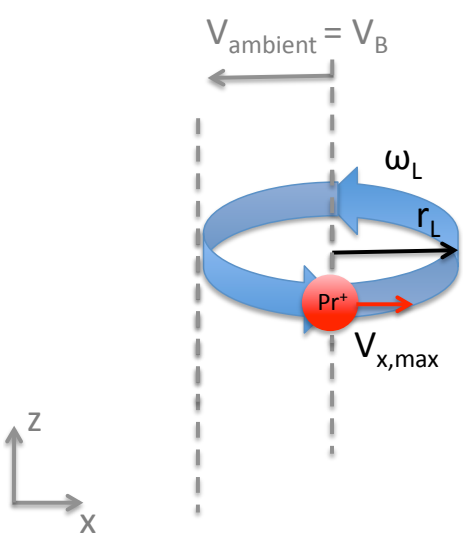

(c) Magnetized $\mathrm{Pr}^{+}$ion on new guiding center.

Figure 2. Schematic of cross-field diffusion mechanism incorporated in magnetic field model for $B=0.5$ Gs. (a) Magnetized $\mathrm{Pr}^{+}$ion with gyroscopic motion about magnetic field line undergoes MEX collision (b) with either $\mathrm{O}$ or Pr. Post-collision velocity of $\mathrm{Pr}^{+}$is used to update the phase angle, which defines new guiding center (c).

The velocity $V_{x 0}^{i o n}$ is the initial velocity of the magnetized ion as it enters the gyro-orbit, which is assumed equal to the post-collision velocity of a $\mathrm{Pr}^{+}$ion formed through CEX. When the ion enters the orbit, the phase angle, $\phi^{i o n}$, is specified according to the relative velocity of the ion with respect to the magnetic field line velocity, $V_{B}$. This is shown schematically in Figure 2(a), for a case in which the ambient flow (and therefore the field line velocity) is directed to the left. If the ion has a velocity to the left relative to the field line, the ion enters the orbit with a phase of $\phi^{i o n}=\pi / 2$. This represents a minimum orbital velocity, and the ion velocity is thus $V_{x 0}=V_{x, \min }$. If the ion has a velocity to the right relative to the field line, the ion enters the orbit with a phase of $\phi^{i o n}=3 \pi / 2$, and the ion velocity corresponds to $V_{x 0}=V_{x, \max }$. The time $t$ in Eq.(6) is initialized to zero when the ion enters the orbit, and is advanced by the simulation timestep. The gyration frequency in Eq.(5) is constant, and has a value $\omega_{L}=233 \mathrm{rev} / \mathrm{s}$. The timestep used in the simulations is $d t=2.5 \times 10^{-4} \mathrm{~s}$, and thus a single orbit is resolved by approximately 17 simulation timesteps.

Once magnetized, the motion of the $\mathrm{Pr}^{+}$ions is a superposition of the magnetic field velocity and the unsteady gyration velocity in the $x$-direction. In a collisional flow, however, these magnetized ions may undergo collisions with other particles. A collision resulting in both momentum and charge exchange would effectively 'demagnetize' the $\mathrm{Pr}^{+}$ion, and the resulting Pr neutral would follow a linear trajectory according to its post-collision velocity. This demagnetization process is modeled for $\mathrm{Pr}^{+}-\mathrm{Pr}$ charge transfer only, as explained in Section B and summarized in Table 1.

It is also possible for a collision between a magnetized $\mathrm{Pr}^{+}$ion and a neutral particle to result in momentum exchange only. Although the $\mathrm{Pr}^{+}$ion keeps its charge and remains magnetized, the momentum exchange will effectively bump the $\mathrm{Pr}^{+}$onto a new orbit with a new guiding center. This process is shown schematically in Figures 2(b) and 2(c), in which a magnetized $\mathrm{Pr}^{+}$ion undergoes a MEX collision with an $\mathrm{O}$-atom. In this particular scenario, the $\mathrm{Pr}^{+}$ion velocity has a phase $\phi^{i o n}=0$, and thus has zero velocity relative to the field line guiding center. Upon collision, the momentum transfer will result in a finite postcollision $x$-velocity component (e.g., to the right as shown by the red arrow). This post-collision velocity defines the initial velocity $V_{x 0}^{\text {ion }}$ for a different orbit about a new guiding center, shown in Figure 2(c). To define the guiding center of the new orbit, the phase angle $\phi^{i o n}$ must be evaluated after each MEX collision using the velocity criteria specified in Eq. (7), where $V_{x 0}^{\text {ion }}$ is taken as the $\mathrm{Pr}^{+}$post-collision velocity.

This treatment of MEX collisions involving magnetized $\mathrm{Pr}^{+}$ions provides a mechanism for cross-field diffusion of the spacecraft ions within these simulations. The cross-field diffusion process is modeled for 
both $\mathrm{Pr}^{+}-\mathrm{Pr}$ and $\mathrm{Pr}^{+}-\mathrm{O}$ momentum exchange events, as summarized in Table 1. As mentioned in Section $\mathrm{B}$, the ambient $\mathrm{O}^{+}$ions are modeled as being trapped indefinitely on the magnetic field lines to preserve the ambient conditions. In consideration of the gyroscopic parameters in Eqs.(4) and (5), the $\mathrm{O}^{+}$ions are modeled as magnetized on field lines of infinite strength, $B \rightarrow \infty$. The influence of the magnetic field on the spacecraft ions, $\mathrm{Pr}^{+}$, is examined in this work by considering the limiting cases of $B \rightarrow \infty, B \rightarrow 0$, and the intermediate case of $B=0.5 \mathrm{Gs}$, found in LEO.

\section{Plume Configurations}

The plume flow examined in this study involves the steady firing of a spacecraft thruster into the ambient ionosphere free stream in Low Earth Orbit. The flow is simulated on an axi-symmetric spherical computational domain shown in Figure 3, with a radius of $22 \mathrm{~km}$ and an axis of symmetry along the $x$-axis. The thruster considered in this study is located at the origin $(x, z)=0$, and generates thrust in the $-x$-direction, such that the plume flow is initially directed in the $+x$-direction (indicated by red arrow in Figure 3 ). An inflow boundary condition is specified at the nozzle exit plane (not visible in Figure 3), with a nominal mass flow rate typical of the Space Shuttle reaction control system (RCS) jets, $\dot{m}=4.2 \mathrm{~kg} / \mathrm{s}$. This mass flow rate is based on the known specific impulse and velocity increment for the RCS jets on a fully-fueled Orbiter $^{18}$ and assuming a simulated nozzle radius of $0.15 \mathrm{~m}$. The plume at the exit plane is modeled as a charge-neutral single species Pr propellant with the properties shown in Table 3 . The orbital motion of the spacecraft thruster is equivalent to an ionosphere free stream velocity in either $\pm x$ direction, indicated by the grey arrow in Figures 3(a), 3(b). The ionosphere freestream is comprised of the neutral $\mathrm{O}$-atoms and single charge $\mathrm{O}^{+}$ions.

The configuration in which the spacecraft thruster fires against the ambient flow is referred to as the ram flow configuration, shown in Figure 3(a). The wake flow configuration refers to the case in which the spacecraft thruster fires with the ambient flow, shown in Figure 3(b). Recalling discussion of the magnetic field model, the magnetic field lines are aligned vertically in these figures, with velocity equal to the ambient velocity. The spacecraft Pr vapor plume expands into the low-density ambient ionosphere and undergoes charge exchange (CEX) collisions, resulting in an ion plume comprised of single-charge $\mathrm{Pr}^{+}$ions.

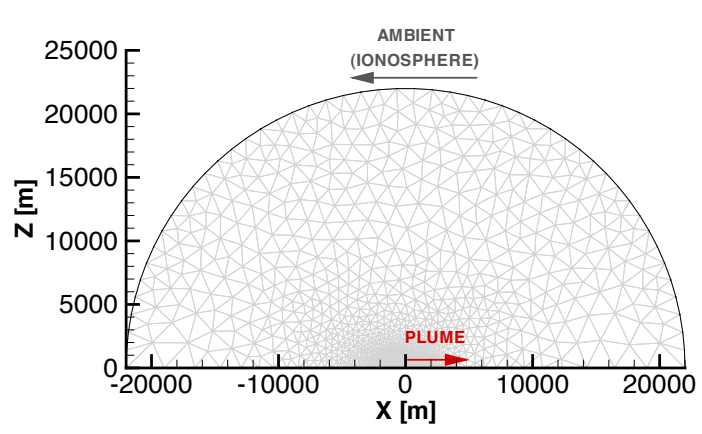

(a) Ram flow

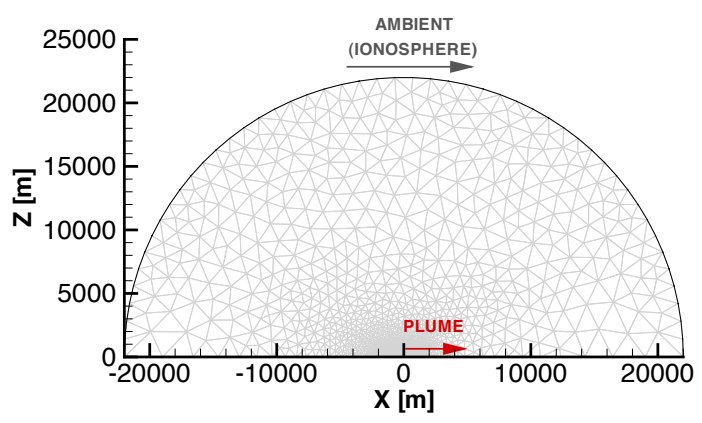

(b) Wake flow

Figure 3. DSMC/PIC computational domain. Axis of symmetry lies along the z-axis, and the nozzle exit plane is located at the origin. Arrows indicate the plume/ambient flow directions for (a) ram flow and (b) wake flow configurations.

Table 3. Plume and Ambient Flow Conditions

\begin{tabular}{|c||c|c|c|c|}
\hline Species & $\mathrm{m}\left[\mathrm{kg} \mathrm{kmol}^{-1}\right]$ & $k_{b} T / q_{o}[\mathrm{eV}]$ & $\mathrm{V}\left[\mathrm{ms}^{-1}\right]$ & $\mathrm{n}\left[\mathrm{m}^{-3}\right]$ \\
\hline $\mathrm{Pr}$ & 20.7 & 0.06 & 2990 & $5.8 \times 10^{23}$ \\
$\mathrm{Pr}^{+}$ & 20.7 & - & - & - \\
$\mathrm{O}$ & 16.0 & 0.06 & \pm 7640 & $9.3 \times 10^{13}$ \\
$\mathrm{O}^{+}$ & 16.0 & 0.06 & \pm 7640 & $1.0 \times 10^{11}$ \\
\hline
\end{tabular}


The properties of the plume at the rocket nozzle and the ambient flow are summarized in Table 3 . The ambient/inflow properties of temperature, velocity and number density are specified only for the CEX reactant species initialized as ambient or at the inflow boundary. Constituents of the ambient ionosphere $\left(O, O^{+}\right)$at an altitude of $400 \mathrm{~km}$ are assigned velocities in the $\pm x$-direction, according to the plume configuration being modeled.

\section{Results}

In this section, steady-state results are presented for both the ram and wake flow configurations. In particular, the influence of the magnetic field on the development of both the spacecraft neutral and ion plumes is investigated. Three magnetic field strengths are considered: $B \rightarrow 0$ and $B \rightarrow \infty$ which are the two limiting cases, as well as an intermediate LEO case in which $B=0.5$ Gs. Recall that only the $\operatorname{Pr}^{+}$ions become magnetized, thus the magnetic field does not directly impact the motion of the neutral Pr particles. It is important to note, however, that the magnetized $\mathrm{Pr}^{+}$ions may become demagnetized through CEX reactions, and so the influence of the magnetic field on the development of the neutral Pr plume is examined as well.

\section{A. Ram Flow Configuration}

The steady-state flow field generated in the ram configuration is presented in Figures $4(\mathrm{a}-\mathrm{c})$. The ambient ionosphere flow in this case is from right to left, opposing the plume flow at the origin. The contours represent the number density of the Pr neutrals, which are emitted from the thruster. As the neutral plume expands outward from the thruster, the Pr particles may undergo MEX and CEX collisions with the ambient particles as well as with each other. The first case shows the development of the neutral plume in the absence of a magnetic field, Figure 4(a). The neutral plume expands outward into the oncoming ambient flow and is largely unaffected by collisions with the relatively low-density ionospheric flow of $\mathrm{O}$ and $\mathrm{O}^{+}$.

Figures 4(b) and 4(c) present the development of the spacecraft neutral plume assuming infinite field strength and field strength $B=0.5$ Gs, respectively. Comparison to Figure 4(a) shows that the magnetic field has a dramatic effect on the development of the neutral Pr plume. The spacecraft neutral particles are emitted from the thruster in the positive $x$-direction, but in both cases, the neutral plume is pushed downstream of the thruster, in the direction of the ambient flow. Since the magnetic field does not directly modify the motion of the Pr particles, this indicates that the majority of spacecraft neutrals emitted from the thruster undergo CEX to form magnetized $\mathrm{Pr}^{+}$ions. These ions are swept downstream by the field lines, but then become demagnetized through a subsequent CEX collision. The details by which the ions are magnetized is found to affect the neutral plume shape. The plume in Figure 4(c) assuming $B=0.5$ Gs appears more diffuse than the plume under infinite field strength in Figure 4(b). The neutral plume is found to persist far upstream of the thruster origin under both $B \rightarrow \infty$ and $B=0.5$ Gs conditions. Under an infinite field strength, the neutral plume propagates $6 \mathrm{~km}$ upstream of the thrusters, with a number density of $O\left(10^{13}\right) \mathrm{m}^{-3}$. Considering the magnetic field strength $B=0.5 \mathrm{Gs}$, the neutral plume with number density $O\left(10^{13}\right) \mathrm{m}^{-3}$ is found to propagate $9 \mathrm{~km}$ upstream of the thrusters, significantly farther than the infinite field strength model.

This difference in both plume shape and upstream propagation of the neutral plume is largely due to the gyroscopic motion of the magnetized ions that is captured in the $B=0.5$ Gs model. Recall that ions become demagnetized through a CEX reaction, resulting in the formation of a neutral particle. According to the $B \rightarrow \infty$ model, the pre-collision velocity of the magnetized ion is equivalent to the orbital velocity, $V_{B}=-7640 \mathrm{~m} / \mathrm{s}$. The differential cross section model suggests that CEX interactions for this system are characterized by a strong forward-scattering tendency, and so the post-collision velocities of demagnetized $\operatorname{Pr}$ particles will generally be in the $-x$-direction. This suggests that most of the Pr neutrals found upstream of the thruster in the $B \rightarrow \infty$ case originate from the thruster and have not yet undergone a CEX collision. Considering the $B=0.5$ Gs model, ions may become demagnetized at any point on the gyroscopic orbit. This implies that ions may have pre-collision velocities in either the $+x$-direction or $-x$-direction, depending on when the CEX collision takes place. Since the CEX interaction is forward scattering, this results in postcollision $\operatorname{Pr}$ velocities in either the $+x$-direction or $-x$-direction, thus enhancing the upstream propagation of the neutral Pr plume.

The steady-state flow field generated by the ram configuration is presented again in Figures $5(\mathrm{a}-\mathrm{c})$, but 


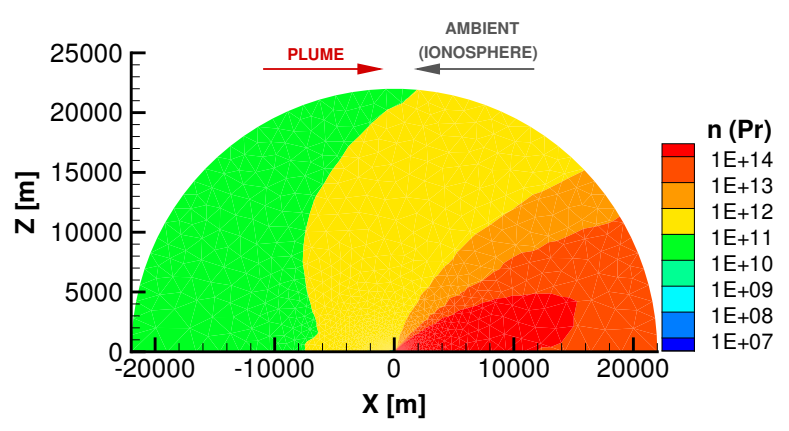

(a) Contours of Pr number density, $B \rightarrow 0$.

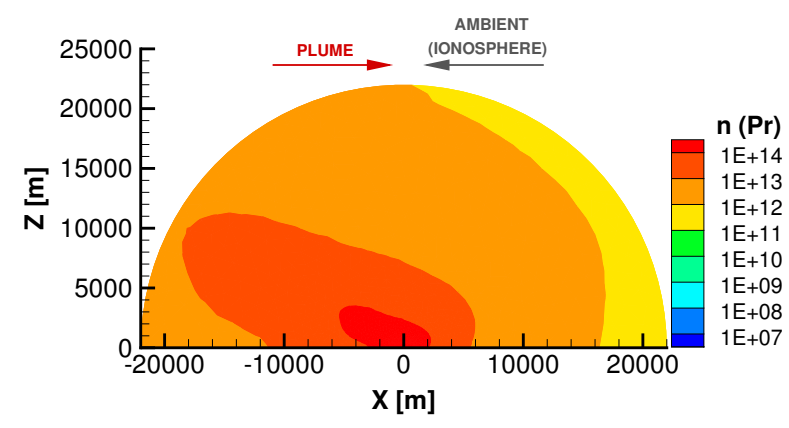

(b) Contours of Pr number density, $B \rightarrow \infty$.

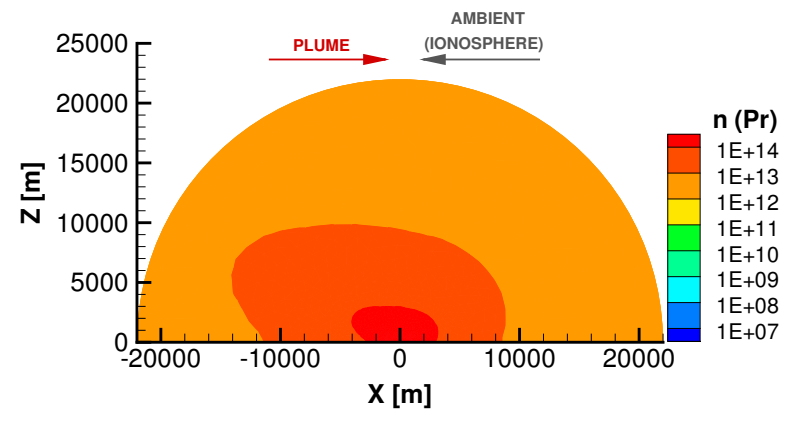

(c) Contours of Pr number density, $B=0.5$ Gs.

Figure 4. Contours of $\operatorname{Pr}$ number density formed in the ram flow configuration, assuming (a) no magnetic field, (b) infinite magnetic field strength, and (c) magnetic field strength at LEO. The magnetic field model accounts for the gyroscopic motion of magnetized ions in the LEO case. 


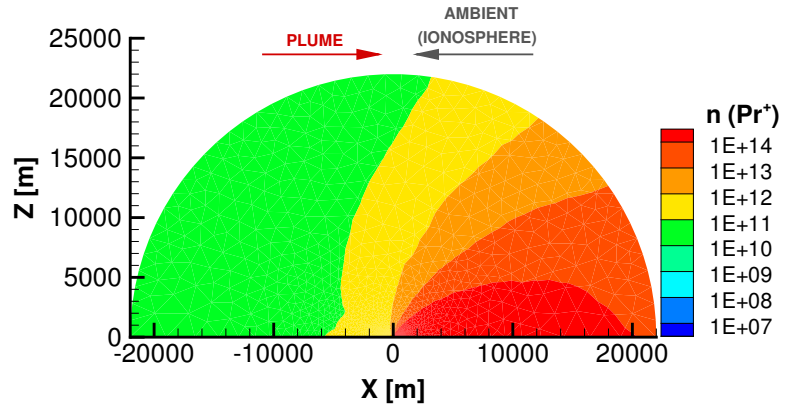

(a) Contours of $\mathrm{Pr}^{+}$number density, $B \rightarrow 0$.

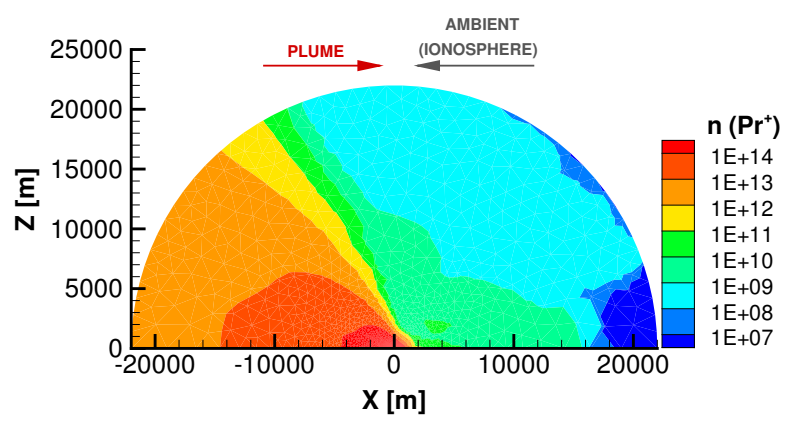

(c) Contours of $\mathrm{Pr}^{+}$number density, $B=0.5$ Gs.

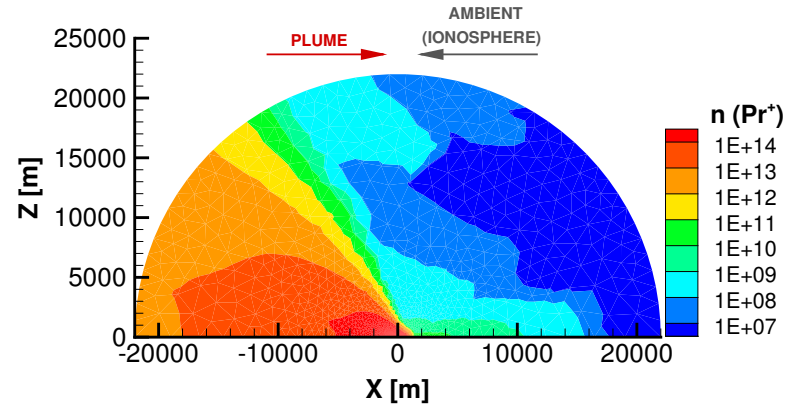

(b) Contours of $\mathrm{Pr}^{+}$number density, $B \rightarrow \infty$.

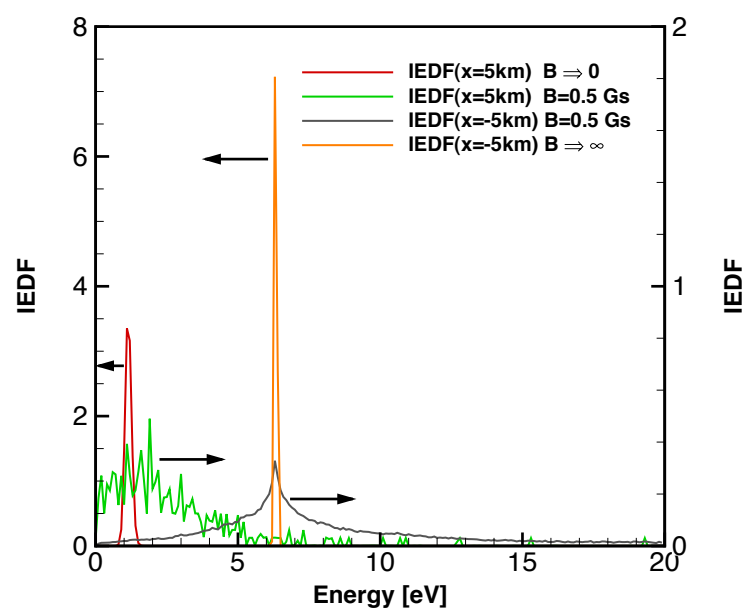

(d) $\mathrm{Pr}^{+}$ion energy distributions sampled $\pm 5 \mathrm{~km}$ of thruster origin for $B \rightarrow 0, B=0.5, B \rightarrow \infty$.

Figure 5. Contours of $\mathrm{Pr}^{+}$number density formed through charge exchange of the spacecraft neutrals, Pr, with the ambient $\mathrm{O}^{+}$ions in the ram flow configuration, assuming (a) no magnetic field, (b) infinite magnetic field strength, and (c) magnetic field strength at LEO. (d) Ion energy distributions sampled $\pm 5 \mathrm{~km}$ from the thruster origin. The IEDFs corresponding to $B=0.5 \mathrm{Gs}$ account for the gyroscopic motion of magnetized ions. 
with focus on the evolution of the ion plume. The contours represent the number density of the $\mathrm{Pr}^{+}$ions, which are generated as a result of charge exchange with the ambient $\mathrm{O}^{+}$ions. Figure 5 (a) shows the $\operatorname{Pr}^{+}$ plume development in the absence of a magnetic field. The high density Pr vapor plume expands outward in the $+x$-direction, at an initial velocity of $2990 \mathrm{~m} / \mathrm{s}$. Pr neutrals that undergo CEX with the ambient $\mathrm{O}^{+}$ have a strong forward-scattering tendency. Thus, it is observed that even with the opposing ambient flow, the ion plume propagates in the $+x$-direction. The contours in Figure $5(\mathrm{~b})$ show the $\mathrm{Pr}^{+}$ion plume in the presence of a magnetic field with infinite strength. In the limit of $B \rightarrow \infty$, the Larmor radius and gyration frequency go to zero and infinity, respectively. Thus, this field strength is modeled by assuming that $\operatorname{Pr}^{+}$ ions formed through CEX become trapped on field lines immediately, with no gyration. The magnetic field is found to have a significant impact on the plume development, as shown in Figure 5(b). $\mathrm{Pr}^{+}$ions are found upstream of the thruster, but these are ions generated from neutral Pr vapor that convects upstream against the ambient ionosphere flow. Once these neutral particles undergo CEX, they are immediately trapped on the field lines, and move in the $-x$-direction with the velocity of the field lines, $-7640 \mathrm{~m} / \mathrm{s}$. Note that although the magnetic field generally constrains the upstream propagation of the ion plume, the $\operatorname{Pr}^{+}$ions are still free to move along the field lines in the $\pm z$-direction.

The intermediate case, $B=0.5 \mathrm{Gs}$, is shown in Figure $5(\mathrm{c})$. $\mathrm{Pr}^{+}$ions that form through CEX are assumed to gyrate about the field lines with frequency $\omega_{L}=233 \mathrm{rev} / \mathrm{s}$, and a Larmor radius determined by the post-collision velocity immediately after the charge exchange collision. For an ion with a velocity of $2990 \mathrm{~m} / \mathrm{s}$ and a field line velocity of $-7640 \mathrm{~m} / \mathrm{s}$, the corresponding Larmor radius is approximately $45 \mathrm{~m}$. The ion plume is qualitatively similar to the $B \rightarrow \infty$ case in Figure 5(b). The ion plume number density downstream of the thruster is slightly higher in the $B \rightarrow \infty$ case.

The ion energy distribution functions (IEDF) of the $\mathrm{Pr}^{+}$ions are compared in Figure 5(d) for the three field strengths. The distributions are sampled at locations $\pm 5 \mathrm{~km}$ from the thruster origin, and $1 \mathrm{~km}$ above the axis of symmetry. The IEDF is determined for only those ions which cross the sampling surface in the radial direction outward from the thruster. In the case of infinite field strength, a trace amount of $\mathrm{Pr}^{+}$ions are generated upstream at the $+5 \mathrm{~km}$ sampling location through charge exchange. These ions, however, are immediately magnetized and swept downstream in the $-x$-direction, and are thus never counted in the IEDF. The IEDF for the $B=0.5$ Gs case is reported at both $\pm 5 \mathrm{~km}$ sampling locations. Similar to the $B \rightarrow \infty$ case, few ions are found at the $5 \mathrm{~km}$ location. However, $\mathrm{Pr}^{+}$that are formed upstream become trapped on field lines, and the gyration motion carries these ions across the sampling region.

Several interesting observations may be made from these energy distributions. It is first noted that the IEDF of $\mathrm{Pr}^{+}$ions sampled $5 \mathrm{~km}$ upstream of the thruster origin is centered over approximately $1 \mathrm{eV}$. This energy corresponds to the initial energy of the thruster Pr particles. Thus, the Pr neutrals suffer little momentum exchange with the oncoming ambient flow, prior to charge exchange. The differential cross section for the $\mathrm{O}^{+}-\mathrm{Pr}$ system produces a strong forward scattering tendency, thus little momentum is transferred during the charge exchange process. This is observed for both the $B \rightarrow 0$ and $B=0.5$ Gs cases. However, the IEDF for $B=0.5$ Gs (shown by the green curve), although centered over $1 \mathrm{eV}$, is very wide in comparison to the peaked IEDF for $B \rightarrow 0$. The effect of the magnetic field is thus to broaden the energy distribution of the magnetized ions, which is consistent with the unsteady gyration motion imposed on the magnetized $\mathrm{Pr}^{+}$ions in the $\mathrm{x}$-direction. A similar trend is found through comparison of the IEDF sampled at $-5 \mathrm{~km}$ from the thruster origin. The IEDF of both $B=0.5$ Gs and infinite field strengths is centered over $6.5 \mathrm{eV}$, which corresponds to the energy of the ambient particles. The IEDF corresponding to the LEO field strength is very broad in comparison to the IEDF of the infinite field strength case.

The influence of the cross-field diffusion is also examined for the $B=0.5$ Gs case. Recalling from Section $\mathrm{C}$, diffusion of the $\mathrm{Pr}^{+}$ions across the magnetic field lines is modeled through MEX collisions, in which new guiding centers are determined based on the post-collision velocity and phase angle of the magnetized ion. To disable this cross-field diffusion, the MEX collisions of $\mathrm{O}-\mathrm{Pr}^{+}$and $\mathrm{Pr}-\mathrm{Pr}^{+}$are processed, but the phase angle is not updated. This subsequently increases/decreases the Larmor radius of the magnetized $\mathrm{Pr}^{+}$ions, but they remain fixed on the same guiding center until they become demagnetized through a CEX reaction. The ram flow simulation is re-computed using this model, and comparisons of the current density with and without cross-field diffusion are presented in Table 4. The current density is reported at locations $\pm 5 \mathrm{~km}$ from the thruster origin. Cross-field diffusion is found to increase the current density, as ion flux is enhanced through this diffusion mechanism. 
Table 4. Current density due to cross-field diffusion of $\mathrm{Pr}^{+}$

\begin{tabular}{|c|c|c|}
\hline & Without cross-field diffusion & With cross-field diffusion \\
\hline$+5 \mathrm{~km}$ & $6.2 \times 10^{-5}\left[\mathrm{~A} / \mathrm{m}^{2}\right]$ & $7.2 \times 10^{-5}\left[\mathrm{~A} / \mathrm{m}^{2}\right](+16 \%)$ \\
$-5 \mathrm{~km}$ & $7.7 \times 10^{-2}\left[\mathrm{~A} / \mathrm{m}^{2}\right]$ & $9.5 \times 10^{-2}\left[\mathrm{~A} / \mathrm{m}^{2}\right](+23 \%)$ \\
\hline
\end{tabular}

\section{B. Wake Flow Configuration}

The steady-state flow field generated in the ram configuration is presented in Figures $6(\mathrm{a}-\mathrm{c})$. The ambient ionosphere flow in this case is from left to right, in the direction of the plume flow at the origin. The contours represent the number density of the neutral spacecraft particles, Pr, which are emitted from the thruster. The first case shows the development of the neutral plume in the absence of a magnetic field, Figure 6(a). The neutral plume expands outward into the ambient co-flow and is again largely unaffected by collisions with the relatively low-density ionospheric flow of $\mathrm{O}$ and $\mathrm{O}^{+}$, even more so than the ram flow configuration shown in Figure 4(a).

Figures 6(b) and 6(c) present the development of the spacecraft neutral plume assuming infinite field strength and field strength $B=0.5 \mathrm{Gs}$, respectively. Comparison to Figure 6 (a) shows that the magnetic field does influence the development of the neutral Pr plume, although the influence is minor compared to the ram flow configuration. The neutral plumes in both cases appear to spread outward and slightly upstream compared to the neutral plume in Figure 6(a), although the direction of plume propagation in Figures 6(b,c) are generally in the $+x$-direction.

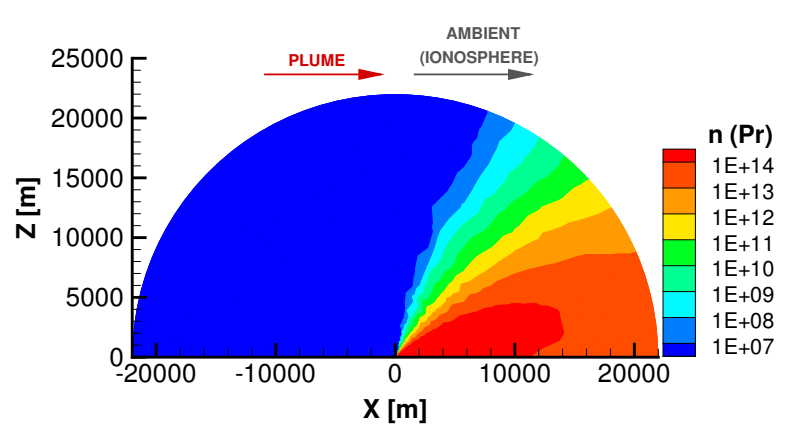

(a) Contours of Pr number density, $B \rightarrow 0$.

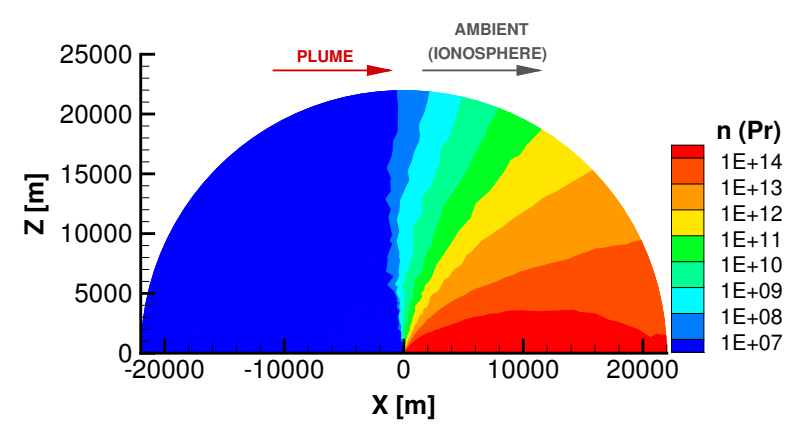

(b) Contours of Pr number density, $B \rightarrow \infty$.

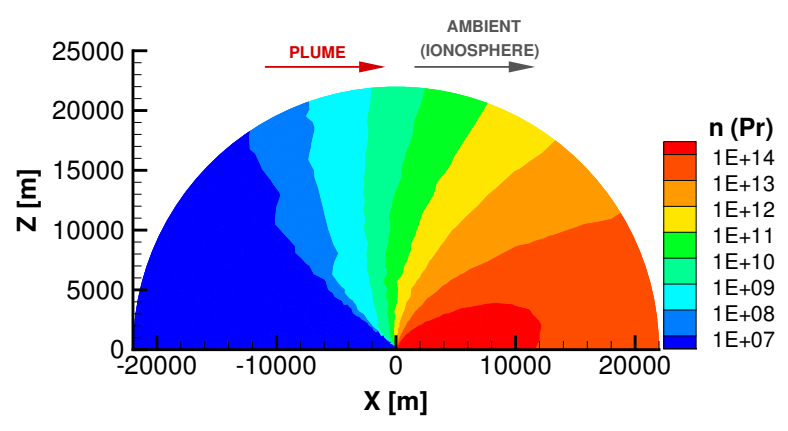

(c) Contours of Pr number density, $B=0.5$ Gs.

Figure 6. Contours of $\operatorname{Pr}$ number density formed in the wake flow configuration, assuming (a) no magnetic field, (b) infinite magnetic field strength, and (c) magnetic field strength at LEO, which accounts for the gyroscopic motion of magnetized ions.

The steady-state flow field generated by the wake configuration is again shown in Figures $7(\mathrm{a}-\mathrm{c})$. Here, the contours represent the number density of the ion species, $\mathrm{Pr}^{+}$. Figure 7(a) shows the ion plume develop- 
ment in the absence of a magnetic field. The plume development is qualitatively very similar to the $B \rightarrow 0$ results from the ram flow configuration, although the ion density upstream is reduced by approximately two order of magnitude, owing to the wake flow configuration. It is interesting to note that although the neutral Pr particles are restricted mostly downstream of the thruster, the ions are found upstream of the thruster in the absence of the magnetic field. The IEDF for this $B \rightarrow 0$ wake flow configuration is nearly identical to the $B \rightarrow 0$ ram flow IEDF. It can be concluded then that the flow direction of the ambient ionosphere relative to the spacecraft plume has very little impact on the ion energy distribution when the magnetic field model is not included.

The contours in Figure 7(b) show the $\mathrm{Pr}^{+}$plume development under a magnetic field of infinite strength. Comparison to the $B \rightarrow \infty$ ram flow configuration in Figure 5(b) shows a dramatic difference in the ion plume development, as the plume is constrained to the $+x$-direction. The spreading of the ion plume in the $+z$-direction (along the field lines) is less pronounced in this case as well. Recalling that the geomagnetic field lines have the same velocity as the ambient flow, the magnetized ions are swept in the direction of the ambient flow, at the orbital velocity. The IEDF for this case is reported only for the $+5 \mathrm{~km}$ location, as the IEDF at the $-5 \mathrm{~km}$ location is not populated. This distribution of $\mathrm{Pr}^{+}$ion energy in the wake flow configuration is nearly identical to the $B \rightarrow \infty$ IEDF from the ram flow configuration. This is consistent with the energy of magnetized ions that have a velocity equivalent to the orbital velocity.

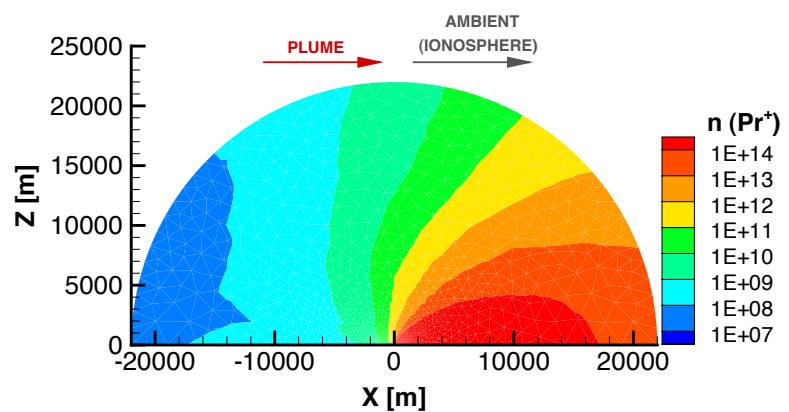

(a) Contours of $\mathrm{Pr}^{+}$number density, $B \rightarrow 0$.

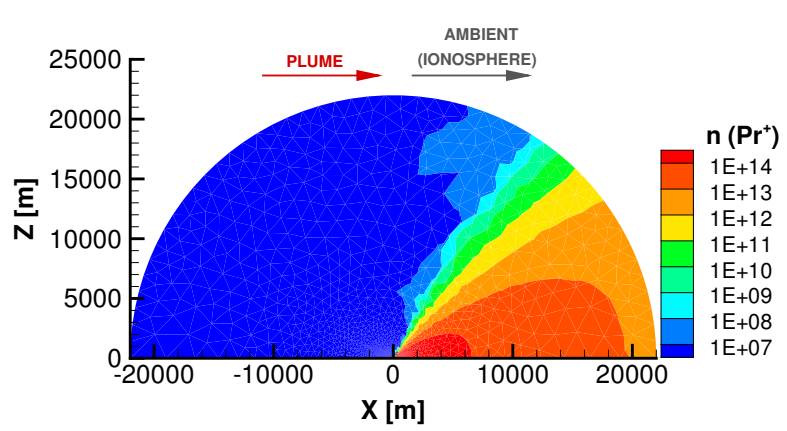

(c) Contours of $\mathrm{Pr}^{+}$number density, $B=0.5$ Gs.

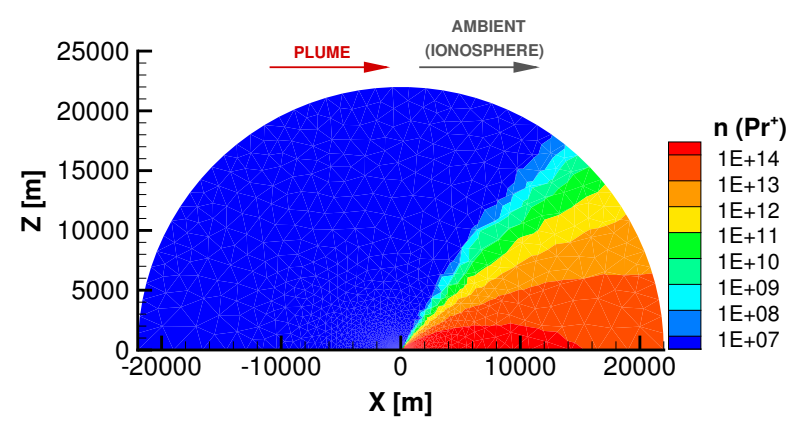

(b) Contours of $\mathrm{Pr}^{+}$number density, $B \rightarrow \infty$.

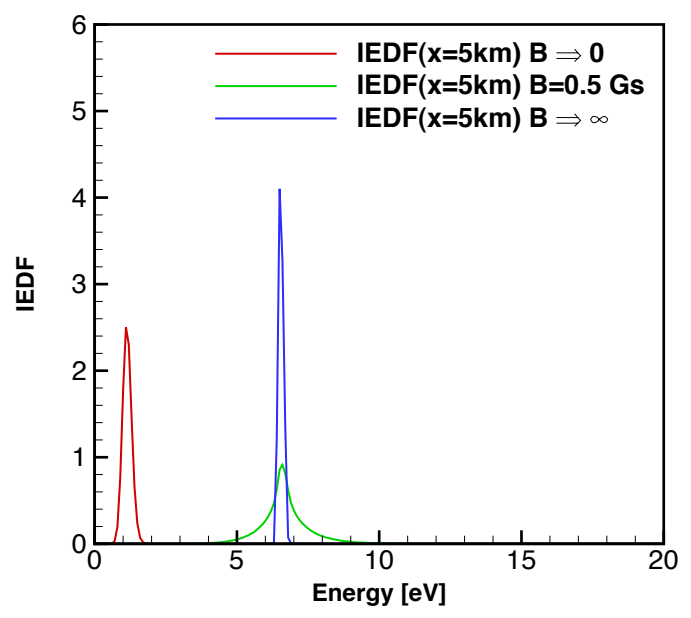

(d) $\mathrm{Pr}^{+}$ion energy distributions sampled $+5 \mathrm{~km}$ of thruster origin for $B \rightarrow 0, B=0.5, B \rightarrow \infty$.

Figure 7. Contours of $\mathrm{Pr}^{+}$number density formed through charge exchange of the spacecraft neutrals, Pr, with the ambient $\mathrm{O}^{+}$ions in the wake flow configuration, assuming (a) no magnetic field, (b) infinite magnetic field strength, and (c) magnetic field strength at LEO. (d) Ion energy distributions sampled $\pm 5 \mathrm{~km}$ from the thruster origin. The IEDFs corresponding to $B=0.5$ Gs account for the gyroscopic motion of magnetized ions.

The development of the ion plume under a magnetic field strength of $B=0.5$ Gs in the wake flow configuration is shown in Figure 5(c). The plume looks qualitatively similar to the plume in Figure 5(b). The IEDF for $B=0.5$ Gs is shown in Figure 7(d), at a location $5 \mathrm{~km}$ from the thruster origin. Again, 
the IEDF is centered over $6 \mathrm{eV}$, which corresponds to the energy of the magnetized ions that travel with the velocity of the magnetic field lines. The IEDF corresponding to $B=0.5$ Gs is again broadened as a result of the gyroscopic motion, although this broadening is more substantial in the ram flow configuration. This is consistent with the fact that the ions that become magnetized in the ram flow configuration have a higher velocity relative to the field lines (approximately $10 \mathrm{~km} / \mathrm{s}$ ) compared to the wake flow configuration (approximately $4 \mathrm{~km} / \mathrm{s}$ ).

\section{Conclusions and Future Work}

The primary focus of this work was to examine the development of a steady spacecraft ion plume formed through charge exchange (CEX) reactions with the ambient ionosphere flow in Low Earth Orbit (LEO). Also of great interest was the influence of the geomagnetic field on the ion plume. To this end, two plume configurations, referred to as ram flow and wake flow, were examined under varying magnetic field strengths. A combined DSMC/PIC methodology was used to properly capture the non-equilibrium collisional and plasma phenomena that are relevant in the rarefied plasma environment in LEO.

The development of the spacecraft neutral and ion plume was first examined in the ram flow configuration, under three magnetic field strengths: two limiting cases, in which $B \rightarrow 0$ and $B \rightarrow \infty$, and $B=0.5$ Gs, which corresponds to the field strength at LEO. The development of both the neutral Pr plume and the $\mathrm{Pr}^{+}$ion plume were strongly influenced by the presence of the magnetic field. The assumption of an infinite field strength provided a reasonable approximation to the more sophisticated model used for $B=0.5$ Gs. The former assumed magnetized ions have a Larmor radius of zero, and are thus trapped on the field lines without a superimposed gyration motion in the $x$-direction. The latter accounted for this finite gyration velocity, and the effect of this gyroscopic motion is to broaden the $\mathrm{Pr}^{+}$IEDF. The impact of cross-field diffusion on the current density was also examined for the $B=0.5$ Gs case, and was found to increase the current density due to an enhancement of $\mathrm{Pr}^{+}$ion flux across field lines.

Similar results were obtained in the wake flow configuration. The ion plumes generated under the three magnetic field strengths were qualitatively similar, as the ambient flow (and thus magnetic field lines) was in the same direction as the plume flow. The observed $\mathrm{Pr}^{+}$IEDFs again demonstrated the broadening of the distribution due to the gyroscopic motion of the magnetized ions.

The assumption of a thruster plume comprised of a single chemical species in this work allowed for a fundamental study of plume/ionosphere interaction in the presence of a geomagnetic field. A more realistic model should include the major chemical reactants of the hypergolic propellant system, which involves the reaction of both fuel (hydrazine) and oxider (nitrogen tetroxide) to produce water vapor, among other products. This will require the use of detailed differential and total cross sections for modeling MEX and CEX interactions among the plume and ionosphere chemical species. Although the present simulations are limited to axisymmetric flows, future work will also examine the evolution of the plume when the plume velocity is aligned with the magnetic field lines.

\section{Acknowledgments}

We gratefully acknowledge the support of Air Force Research Laboratory (AFRL) Space Vehicles Directorate and Barron Associates, Inc. Work was performed under subcontract to Barron Associates, Inc. under AFRL contract FA9453-11-C-0181. Computational resources and technical support were provided by the Center for Advanced Computing at the University of Michigan.

\section{References}

\footnotetext{
${ }^{1}$ Burke, W., Gentile, L., Machuzak, J., Hardy, D., and Hunton, D., "Energy distributions of thruster pickup ions detected by the Shuttle Potential and Return Electron Experiments during TSS 1," Journal of Geophysical Research, 100 A10, 1995.

${ }^{2}$ McMahon, W., Salter, R., Hills, R., and Delorey, D., "Measured electron contribution to shuttle plasma environment," AIAA-83-2598 AIAA Shuttle Environment and Operations Meeting, 1983.

${ }^{3}$ Karabadzhak, G., Plastinin, Y., Khmelinin, B., Teslenko, V., Shvets, N., Drakes, J., Swann, D., and McGregor, W., "Experimentation Using the Mir Station as a Space Laboratory," AIAA Aerospace Sciences Meeting, 1997, pp. AIAA-97-0288.

${ }^{4}$ Drakes, J. and Swann, D., "DSMC Computations of the Progress-M Spacecraft Retrofiring Exhaust Plume," AIAA Aerospace Sciences Meeting, January Reno, NV 1999, pp. AIAA-99-0975.

${ }^{5}$ Kaplan, C. and Bernhardt, P., "Effect of an Altitude-Dependent Background Atmosphere on Shuttle Plumes," Journal
} 
of Spacecraft and Rockets, Vol 47, No 4, July-August 2010.

${ }^{6}$ Bernhardt, P., Ballenthin, J., Baumgardner, J., Bhatt, A., Boyd, I., Burt, J., Caton, R., Coster, A., Erickson, P., Huba, J., Earle, G., Kaplan, C., Foster, J., Groves, K., Haaser, R., Hellis, R., Hunton, D., Hysell, D., Klenzing, J., Larsen, M., Lind, F., Pedersen, T., Pfaff, R., Stoneback, R., Roddy, P., Rodriguez, S., San Antonio, G., Schuck, P., Siefring, C., Selcher, C., Smith, S., Talaat, E.R. an Thomason, J., Tsunoda, R., and Varney, R., "Ground and Space-Based Measurement of Rocket Engine Burns in the Ionosphere," IEEE Transactions on Plasma Science, Vol 40, No 5, May 2012.

${ }^{7}$ Bird, G., Molecular Gas Dynamics and the Direct Simulation of Gas Flows, Oxford University Press, Oxford, 1994.

${ }^{8}$ Birdsall, C. and Langdon, A., Plasma Physics via Computer Simulation, Taylor and Francis, New York, 2004.

${ }^{9}$ Cai, C., Theoretical and Numerical Studies of Plume Flows in Vacuum Chambers, Ph.D. Dissertation, University of Michigan, Ann Arbor, MI, 2005.

${ }^{10}$ Boyd, I. and Dressler, R., "Far field modeling of the plasma plume of a Hall thruster," Journal of Applied Physics, 92 4, 2002.

${ }^{11}$ Pullins, S., Chiu, Y., Levandier, D., and Dressler, R., "Ion dynamics in Hall effect and ion thrusters $-\mathrm{Xe}(+)+\mathrm{Xe}$ symmetric charge transfer," AIAA-00-0603 38th AIAA Aerospace Sciences Meeting, 2000.

${ }^{12}$ Miller, S., Pullins, S., Levandier, D., Chiu, Y., and Dresler, R., "Xenon charge exchange cross sections for electrostatic thruster models," Journal of Applied Physics, 91 3, 2002.

${ }^{13}$ Lindsay, B., Rejoub, R., Sieglaff, D., and Stebbings, R., "Charge transfer of keV O+ ions with CO and H2O," J. Phys. B: At. Mol. Opt. Phys., 34 2159, 2001.

${ }^{14}$ Katz, I., Jongeward, G., Davis, V., Mandell, M., Mikellides, I., Dressler, R., Boyd, I., Kannenberg, K., Pollard, J., and King, D., "A Hall effect thruster plume model including large-angle elastic scattering," AIAA-2001-3355 37th AIAA/ASME/SAI/ASEE Joint Propulsion Conference and Exhibit, 2001.

${ }^{15}$ Turner, B. and Rutherford, J., "Charge transfer and ion-atom interchange reactions of water vapor ions," J. Geophys. Res., 73 6751-6758, 1968.

${ }^{16}$ Dressler, R., Salter, R., and Murad, E., "Dynamics of the O+ H2O charge-transfer reaction and its implications for space-borne measurements," Planet. Space Sci., 40 1695-1704, 1992.

${ }^{17} \mathrm{Li}$, X., Huang, Y.-L., Flesch, G., and Ng, C., "bsolute total cross sections for the ionmolecule reaction O+(4So)+H2O," J. Chem. Phys., 102 5100, 1995.

${ }^{18}$ Stuit, T., "Designing the STS-134 Re-Rendezvous: A Preparation for Future Crewed Rendezvous Missions," AIAA Paper No. 2011-7189, 2011 